\title{
Vaspin attenuates the progression of atherosclerosis by inhibiting ER stress-induced macrophage apoptosis in $\operatorname{apoE}^{-/ 2}$ mice
}

\author{
YING LIN, JIANHUI ZHUANG, HAILING LI, GUOFU ZHU, SHUNPING ZHOU, \\ WEIMING LI, WENHUI PENG and YAWEI XU \\ Department of Cardiology, Shanghai Tenth People's Hospital, Tongji University, \\ School of Medicine, Shanghai 200072, P.R. China
}

Received January 21, 2015; Accepted November 6, 2015

DOI: $10.3892 / \mathrm{mmr} .2015 .4708$

\begin{abstract}
Visceral adipose tissue-derived serine protease inhibitor (vaspin) is a novel adipokine with potential insulin-sensitizing properties, which was initially detected in the visceral adipose tissue of genetically obese rats. Previous studies have demonstrated that vaspin exerts a protective effect on arteries undergoing atherosclerosis in vitro, and it has been shown to exert anti-inflammatory and antimigratory effects on vascular smooth muscle cells. Vaspin promotes proliferation and inhibits apoptosis in endothelial cells, and decreases proliferation of the arterial intima under diabetic conditions. In addition, macrophage apoptosis is an important characteristic of atherosclerotic plaque development. In vivo experiments were performed by histological analysis, including Oil Red O, hematoxylin and eosin and Masson's trichrome staining. Mice were injected with lentivirus via the tail vein and tissues were obtained for histological analysis. Cell apoptosis was determined by flow cytometry of Annexin-V/propidium iodide dual staining and terminal deoxynucleotidyl transferase deoxyuridine triphosphate nick end labeling assay. Total proteins were extracted and protein expression levels were detected by western blot analysis. The present study aimed to investigate whether vaspin was able to protect against atherosclerotic development in vivo, and to explore the underlying mechanisms of the potential antiatherogenic effects. The results of the current study indicated that vaspin inhibited the progression of atherosclerotic plaques in $\mathrm{apoE}^{-/-}$mice by inhibiting endoplasmic reticulum stress-induced macrophage apoptosis.
\end{abstract}

Correspondence to: Professor Yawei Xu or Professor Wenhui Peng, Department of Cardiology, Shanghai Tenth People's Hospital, Tongji University, School of Medicine, 301 Middle Yanchang Road, Shanghai 200072, P.R. China

E-mail: xuyawei@tongji.edu.cn

E-mail: pwenhui@tongji.edu.cn

Key words: endoplasmic reticulum stress, vaspin, macrophages, apoptosis, atherosclerosis

\section{Introduction}

Visceral adipose tissue-derived serine protease inhibitor (vaspin) was initially detected in the visceral adipose tissue of Otsuka Long-Evans Tokushima Fatty rats (1). Vaspin is a compensatory adipokine with anti-inflammatory properties that is able to improve insulin sensitivity, as demonstrated by the administration of recombinant human vaspin protein to diet-induced obese mice $(1,2)$. In addition, elevated serum vaspin concentrations have been detected in humans with obesity, type 2 diabetes and polycystic ovary syndrome, and a correlation has been identified between vaspin and $\mathrm{C}$-reactive protein levels (3-7).

In addition to its ability to ameliorate diabetes, vaspin exerts protective effects on heart disease. For example, low vaspin concentrations correlate with coronary artery disease (CAD) severity and unstable angina pectoris (8). Previous studies have also demonstrated that vaspin may inhibit endothelial cell apoptosis and attenuate high glucose-stimulated vascular smooth muscle cell (VSMC) proliferation and chemokinesis $(9,10)$. Furthermore, vaspin has been reported to stimulate the 78-kDa glucose-regulated protein (GRP78)/murine tumor cell DnaJ-like protein 1 (MTJ-1) complex and subsequent signals, exerting beneficial effects on endoplasmic reticulum (ER) stress-induced metabolic dysfunction (11). In the cardiovascular system, vaspin acts as a ligand for the cell-surface GRP78/voltage-dependent anion channel (VDAC) complex in endothelial cells, promotes proliferation, inhibits apoptosis, and protects against diabetes mellitus-associated vascular injury (12).

Macrophage apoptosis is an important characteristic of atherosclerotic plaque development. Particularly in advanced lesions, macrophage apoptosis is a key factor that aggravates advanced plaque necrosis (13). ER stress has emerged as a general mediator of vascular inflammation and endothelial dysfunction in atherosclerosis, and has been reported to contribute to plaque vulnerability via the induction of macrophage and VSMC apoptosis (14). ER is the major cellular site for protein folding and trafficking, and is central to numerous cellular functions. Failure of the adaptive capacity of ER results in activation of the unfolded protein response, which can lead to cell pathology and subsequent tissue dysfunction (15). In pathological settings, prolonged ER 
stress may trigger apoptosis, either through the functions of inositol-requiring enzyme-1 at the ER or through downstream effectors, including $\mathrm{C} / \mathrm{EBP}$-homologous protein (CHOP). Activation of ER stress contributes to macrophage death and subsequent plaque necrosis in advanced atheroma, a process that has widely been accepted as an important factor in atherosclerosis (16).

It has previously been demonstrated that low vaspin serum concentrations are correlated with recent ischemic events in patients with carotid stenosis (17). In addition, decreased vaspin serum levels have been detected in asymptomatic patients with CAD (18). Furthermore, Zhang et al also demonstrated that low vaspin concentrations are correlated with the severity of CAD and acute coronary diseases (19).

The present study aimed to determine whether the effects of vaspin on plaque progression and morphology in apolipoprotein $\mathrm{E}$ (apoE $)^{-/-}$mice were mediated by inhibition of ER stress-induced apoptotic pathways. In vitro experiments were also performed to examine the role of vaspin in THP-1 macrophages.

\section{Materials and methods}

Ethics statement. The experiments outlined in the present study conform to the Guide for the Care and Use of Laboratory Animals published by the National Institutes of Health (20). All animal procedures were approved by the Animal Care and Use Committees of the Shanghai Tenth People's Hospital (Shanghai, China).

Lentivirus preparation. The vaspin-encoding lentivirus (LV5-mus-vaspin) and control lentivirus (LV5NC) were purchased from Shanghai GenePharma Co., Ltd. (Shanghai, China). The lentiviruses also expressed green fluorescent protein (GFP). Final titers were $1 \times 10^{8} \mathrm{TU} / \mathrm{ml}$ for LV5-mus-vaspin and $1 \times 10^{9} \mathrm{TU} / \mathrm{ml}$ for LV5NC. All experiments described in the present study were performed using the same lentiviruses.

Experimental animals. Male apoE ${ }^{-/-}$mice (age, 6 weeks; $\mathrm{n}=16$ ) and male C57BL/6 mice (age, 6 weeks; n=8) were obtained from Beijing Vital River Laboratory Animal Technology Co., Ltd. (Beijing, China) and fed a high cholesterol diet containing $16.6 \%$ fat, $10.6 \%$ sucrose and $1.3 \%$ cholesterol (Shanghai SLAC Laboratory Animal Co., Ltd., Shanghai, China) for 12 weeks. The mice were housed in cages with a 12-h light/dark cycle. The temperature of the animal room was maintained at $23 \pm 2^{\circ} \mathrm{C}$ and the relative humidity was maintained at $55 \pm 15 \%$. All of the animals were provided free access to drinking water and food. The mice were divided into two treatment groups, $\mathrm{LV} 5 \mathrm{NC}(\mathrm{n}=8)$ and $\mathrm{LV}$-mus-vaspin $(\mathrm{n}=8)$. Each mouse was injected with $200 \mu$ l lentivirus through the tail vein. Body weight and food intake were monitored every 5 days in both groups.

Plasma glucose and lipid panel analysis. Following a 12-h fast and the induction of anesthesia with $3 \%$ pentobarbital (30 mg/kg; China National Medicines Corporation, Ltd., Shanghai, China), plasma samples $(250 \mu \mathrm{l})$ were obtained from all apoE $\mathrm{E}^{-/-}$mice. Fasting glucose, total cholesterol and triglyceride, low-density lipoprotein cholesterol (LDL-C), and high-density lipoprotein cholesterol (HDL-C) levels were measured using a colorimetric enzymatic assay system (Roche Modular P-800; Roche Diagnostics, Basel, Switzerland).

Histological analysis. Following 12 weeks of treatment, at the age of 18 weeks, the animals were sacrificed by euthanasia with an intraperitoneal injection of $3 \%$ pentobarbital at a concentration of $70 \mathrm{mg} / \mathrm{kg}$. The heart and aorta were removed following in situ perfusion with phosphate-buffered saline (PBS). The tissue samples were fixed in $4 \%$ paraformaldehyde for 1 day, and 4- $\mu \mathrm{m}$ paraffin-embedded cross-sections of the aortic root were prepared for the subsequent histological and immunohistochemical analyses. The aortic arch and abdominal aorta were stained en face with Oil Red O (60\% saturated Oil Red O in $40 \%$ deionized water; Sigma-Aldrich, St. Louis, MO, USA), in order to measure lesion development. The cross-sections were stained with hematoxylin and eosin kit (1.5\% hematoxylin and $1 \%$ eosin; Beyotime Institute of Biotechnology, Haimen, China) and Masson's trichrome (Sigma-Aldrich) containing Biebrich scarlet-acid fuchsin solution $(0.9 \%$ biebrich scarlet, $0.1 \%$ acid fuchsin and $1 \%$ acetic acid), $10 \%$ phosphotungstic acid solution, $10 \%$ phosphomolybdic acid solution and aniline blue solution (2.4\% aniline blue and $2 \%$ acetic acid), in order to evaluate atherosclerotic lesion complexity and the percentage of plaque size and necrotic core size. All the experiments were performed according to the manufacturer's protocols. Images of staining were captured using a Leica DMI6000 microscope (Leica Microsystems, Wetzlar, Germany). The percentages were quantified using Image-Pro Plus 6.0 (Media Cybernetics, Inc., Rockville, MD, USA).

Immunohistochemical analysis. The tissue cross-sections were labeled with mouse monoclonal anti-CHOP (1:300; Cell Signaling Technology, Inc., Danvers, MA, USA; cat. no. 2895), mouse monoclonal anti-GFP (1:300; Cell Signaling Technology; cat. no. 2955), and goat monoclonal anti-vaspin (1:200; Santa Cruz Biotechnology, Inc., Dallas, TX, USA; cat. no. sc-79815) at $4^{\circ} \mathrm{C}$ overnight, following microwave antigen retrieval in citrate buffer. After washing with PBS with Tween 20 (PBST), the tissue samples were incubated with rabbit anti-mouse (1:200; Gene Tech Biotechnology Co., Ltd., Shanghai, China; cat. no. GK500705) and donkey anti-goat secondary antibodies (1:200; Santa Cruz Biotechnology, Inc.; cat. no. sc-2042) at $37^{\circ} \mathrm{C}$ for $1 \mathrm{~h}$, and then incubated with diaminobenzidine substrate (0.05\%; Sigma-Aldrich) for 1-3 min. Specimens were counterstained with hematoxylin and images were captured under a Leica DMI6000 microscope. Three sections from each animal were stained for lesion quantification, which was expressed as a percentage of total lesion area. Data are presented as the mean \pm standard error of the mean (SEM).

Cell culture. Human monocytic THP-1 cells were purchased from the Institute of Biochemistry and Cell Biology (Shanghai, China). The cells were cultured in RPMI-1640 medium (Gibco; Thermo Fisher Scientific, Inc., Waltham, MA, USA) supplemented with $2 \mathrm{mM}$ L-glutamine (Gibco; Thermo Fisher Scientific, Inc.) and $10 \%$ fetal bovine serum (BSA; Gibco; Thermo Fisher Scientific, Inc.) at $37^{\circ} \mathrm{C}$ with $5 \% \mathrm{CO}_{2}$ in a humidified atmosphere (21). The THP-1 cells 
were incubated with $100 \mathrm{nM}$ phorbol-12-myristate 13-acetate (PMA; Sigma-Aldrich) for $48 \mathrm{~h}$ and allowed to differentiate into macrophages. Prior to incubation with oxidized (ox)-LDL (50 $\mu \mathrm{g} / \mathrm{ml}$; Guangzhou Yiyuan Biotech., Co., Ltd., Guangzhou, China) for 24 or $48 \mathrm{~h}$, the PMA-induced macrophages were treated with vaspin (100 ng/ml; Sigma-Aldrich) for $24 \mathrm{~h}$ and harvested for subsequent experiments.

Flow cytometry (FCM) analysis. Apoptosis was evaluated in three independent experiments using an Annexin V Apoptosis Detection kit (Roche Diagnostics Operations, Indianapolis, IN, USA). Macrophages ( $1 \times 10^{5}$ cells/well) were pre-treated with vaspin $(100 \mathrm{ng} / \mathrm{ml})$ for $24 \mathrm{~h}$ and then incubated with ox-LDL $(50 \mu \mathrm{g} / \mathrm{ml})$ for 24 or $48 \mathrm{~h}$. The cells were then harvested and resuspended in binding buffer, followed by double staining with fluorescein isothiocyanate-conjugated Annexin V $(20 \mu \mathrm{g} / \mathrm{ml} ; 5 \mu \mathrm{l})$ and propidium iodide $(50 \mu \mathrm{g} / \mathrm{ml} ; 5 \mu \mathrm{l})$ for $15 \mathrm{~min}$ at $20^{\circ} \mathrm{C}$, in $\mathrm{Ca}^{2+}$-enriched binding buffer. Fluorescence was detected by FACScan flow cytometer (BD Biosciences, Franklin Lakes, NJ, USA).

Terminal deoxyribonucleotide transferase-mediated deoxyuridine triphosphate nick-end labeling (TUNEL) assay. The TUNEL assay was performed using an In Situ Cell Death Detection kit (Roche Diagnostics, Mannheim, Germany). Macrophages were pretreated with vaspin $(100 \mathrm{ng} / \mathrm{ml})$ for $24 \mathrm{~h}$ and then stimulated with ox-LDL $(50 \mu \mathrm{g} / \mathrm{ml})$ for $48 \mathrm{~h}$. The macrophages were subsequently fixed with $4 \%$ paraformaldehyde for $25 \mathrm{~min}$, followed by permeabilization with $0.2 \%$ Triton X-100 for $5 \mathrm{~min}$. Finally, the macrophages were incubated with TUNEL reaction mixture for $1 \mathrm{~h}$ at $37^{\circ} \mathrm{C}$ in the dark, and stained with Hoechst 33342 (Invitrogen; Thermo Fisher Scientific, Inc.) for $20 \mathrm{~min}$. The fluorescence images were obtained using a fluorescence microscope (Leica DMI6000; magnification, x50). The number of TUNEL-positive cells was expressed as a percentage of positive cells double-stained with TUNEL and Hoechst 33342.

Protein extraction and western blot analysis. Protein extraction and western blot analysis were performed as described in our previous study (21). Cells were lysed with ice-cold radioimmunoprecipitation assay buffer (Santa Cruz Biotechnology, Inc.) and centrifuged at $10,000 \mathrm{x} \mathrm{g}$ for $5 \mathrm{~min}$ at $4^{\circ} \mathrm{C}$. Protein concentrations in the supernatants were measured using a Bicinchoninc Acid Protein Assay kit (Pierce Biotechnology, Inc., Rockford, IL, USA). Briefly, protein samples (20 $\mu \mathrm{g})$ were separated by 10 or $12 \%$ sodium dodecyl sulfate-polyacrylamide gel electrophoresis and transferred to nitrocellulose membranes (CW Biotech, Beijing, China). The membranes were blocked with $5 \% \mathrm{BSA}$ for $1 \mathrm{~h}$ and were then incubated with the following antibodies at $4^{\circ} \mathrm{C}$ overnight: Anti-activating transcription factor (ATF)6 (1:1,000; ProteinTech Group, Inc., Chicago, IL, USA; cat. no. 24169-AP), anti-CHOP (1:1,000), anti-total (t)-c-Jun N-terminal kinases (JNK)1/2 (1:800; Cell Signaling Technology, Inc., cat. no. 9258, anti-phosphorylated (p)-JNK1/2 (1:800; Cell Signaling Technology, Inc.; cat. no. 4671), anti-cleaved-caspase 12 (1:800; BioVision, Inc., Milpitas, CA, USA; cat. no. 3282-100), anti-cleaved-caspase 9 (1:800; Abcam, Cambridge, MA, USA; cat. no. ab2325) and anti-cleaved-caspase 3 (1:800; Abcam; cat. no. ab2302).
Anti-b-actin served as the control antibody $(1: 5,000$; Shanghai Yeasen Biotechnology Co., Ltd., Shanghai, China; cat. no. 30101ES50) The membranes were then washed with PBST and incubated with horseradish peroxidase-conjugated anti-rabbit immunoglobulin $\mathrm{G}$ ( $\mathrm{IgG}$ ) secondary antibody (1:2,000; GE Healthcare Life Sciences, Chalfont, UK; cat. no. NA9310) or anti-mouse IgG secondary antibodies (1:2,000; GE Healthcare Life Sciences; NA931) for $1 \mathrm{~h}$, and were visualized using an Odyssey Imaging system (LI-COR Biosciences, Lincoln, NE, USA).

Statistical analysis. All statistical analyses were performed using SPSS 14.0 (SPSS, Inc., Chicago, IL, USA). Data are presented as the mean \pm SEM. Differences between two groups were examined using Student's t-test. One-way analysis of variance was used to compare multiple groups, if appropriate, with Bonferroni correction for the post-hoc analysis. $\mathrm{P}<0.05$ was considered to indicate a statistically significant difference, and $\mathrm{P} \leq 0.016$ was considered to indicate a statistically significant difference for post-hoc analysis. All experiments were performed at least three times.

\section{Results}

Vaspin inhibits the progression of atherosclerosis in apoE ${ }^{-/}$ mice. Body weight and food intake of the apoE $\mathrm{E}^{-/-}$mice were not influenced by LV5-mus-vaspin transfection during the 12-week feeding regimen (Fig. 1A). There was no difference between the LV5NC and LV5-mus-vaspin-transfected groups in circulating levels of triglyceride, LDL-C, HDL-C and glucose; however, the total cholesterol level was markedly decreased in the LV5-mus-vaspin-transfected mice (27.12 \pm 1.39 vs. $33.04 \pm 1.70 \mathrm{mmol} / \mathrm{l} ; \mathrm{P}<0.001$; $\mathrm{n}=8$; Fig. 1B). Whole aortas, from the ascending aorta to the abdominal aorta, were cut open, periaortic fat and blood clots were removed, and the vessels were longitudinally sectioned and stained with Oil Red O, in order to detect lipid deposition. The lesions were then measured as a percentage of plaque area. As shown in Fig. 1C, LV5-mus-vaspin-transfected apoE ${ }^{-/}$ mice exhibited significantly reduced lesion development, as compared with the LV5NC-transfected group (plaque area $11.03 \pm 1.34$ vs. $34.74 \pm 1.98 \% ; \mathrm{P}<0.001 ; \mathrm{n}=5$ ).

To confirm that the apoE $\mathrm{E}^{-/-}$mice were successfully transfected with the lentiviruses, immunohistochemical staining of GFP and vaspin was conducted in the aortic sinus of the C57BL/6 mice, LV5NC-transfected apoE ${ }^{-/-}$mice (negative control), and LV5-mus-vaspin-transfected mice. As shown in Fig. 2C, the expression of GFP was higher in the LV5-mus-vaspin-transfected apoE ${ }^{-/-}$mice, as compared with the $\mathrm{C} 57 \mathrm{BL} / 6$ mice. In addition, the expression of vaspin was increased in the LV5-mus-vaspin-transfected apoE $\mathrm{E}^{-/-}$mice, as compared with the LV5NC-transfected group and C57BL/6 mice.

Vaspin decreases atherosclerotic plaque size and attenuates ER stress in apoE ${ }^{-/}$mice. Masson's trichrome staining was used to evaluate plaque stabilization. Vaspin markedly enhanced collagen content, whereas the lipid-rich necrotic core size was significantly decreased following vaspin transfection $(\mathrm{P}<0.01$; Fig. 2A). Considering other aspects of plaque composition, 


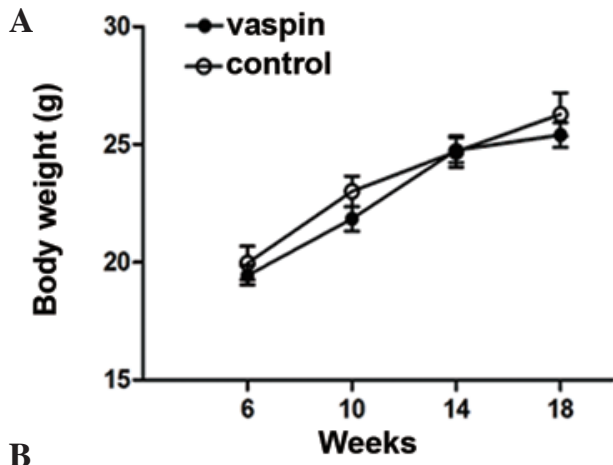

B

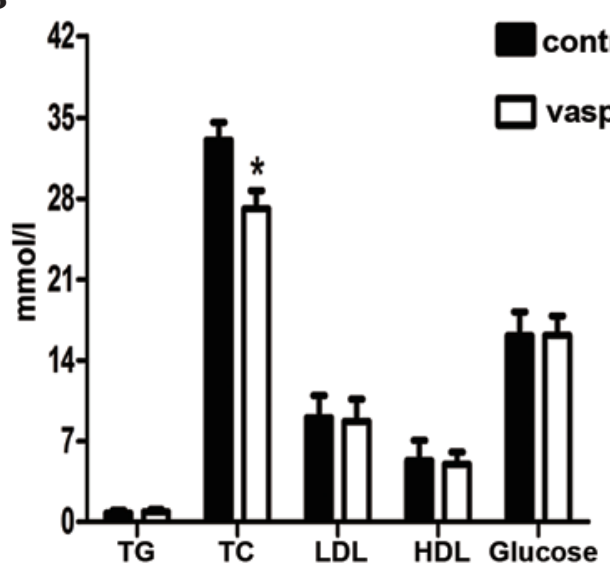

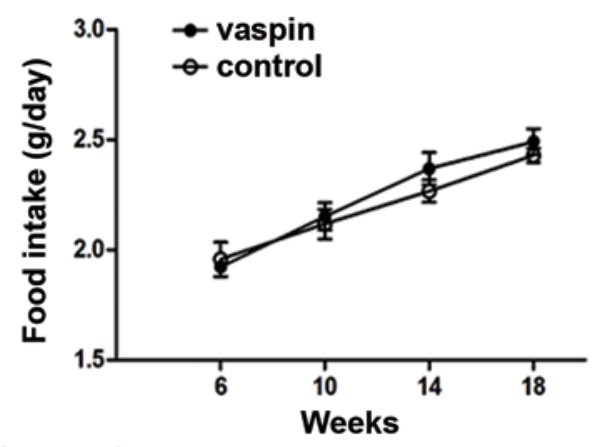

C
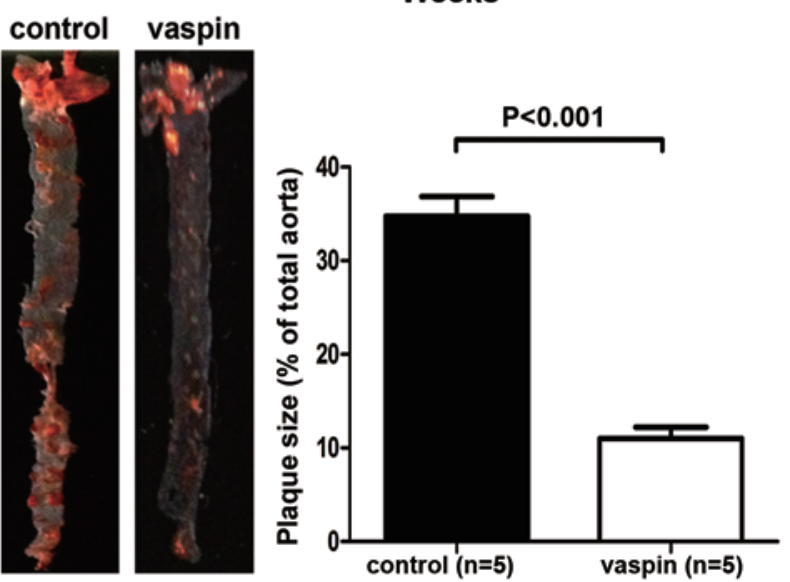

Figure 1. Visceral adipose tissue-derived serine protease inhibitor (vaspin) inhibits the development of atherosclerosis in apolipoprotein $\mathrm{E}$ (apoE) ${ }^{-/}$mice. (A) Comparisons of body weight and food intake in $\mathrm{apoE}^{-/}$mice injected with control lentivirus and vaspin-encoding lentivirus at different ages (200 $\mu 1$ each). (B) Plasma levels of total triglyceride (TG), total cholesterol (TC), low-density lipoprotein cholesterol (LDL), high-density lipoprotein cholesterol (HDL) and glucose after 12 weeks of high cholesterol diet. (C) En face plaque quantification of the aortic arch and thoracic aorta stained with Oil Red O. The data in (A and B) were obtained from eight mice from each group. The data in (C) were obtained from five mice from each group. Data are presented as the mean \pm standard error of the mean. ${ }^{*} \mathrm{P}<0.001$ compared with the control group.

an immunohistochemical analysis detected reduced $\mathrm{CHOP}$ expression in the aortic sinus of LV5-mus-vaspin-transfected apoE $^{-/-}$mice $(\mathrm{P}<0.001$; Fig. 2B).

Vaspin inhibits ER stress-induced apoptosis in macrophages. To confirm the inhibitory effects of vaspin on ER stress-induced apoptosis, Annexin V/propidium iodide dual staining and FCM, and TUNEL analyses were performed. Representative images from the FCM assays and summarized data are shown in Fig. 3A. As compared with the control group, ox-LDL over 24 and $48 \mathrm{~h}$ significantly increased rates of apoptosis $(0.73 \pm 0.06$ vs. $14.10 \pm 1.57 \%$; $\mathrm{P}<0.001 ; 0.73 \pm 0.06$ vs. $18.73 \pm 1.53 \% ; \mathrm{P}<0.001$, respectively). Pre-treatment with vaspin $(100 \mathrm{ng} / \mathrm{ml})$ significantly reduced the percentage of late and early apoptotic macrophages $(14.10 \pm 1.57$ vs. $9.77 \pm 1.90 \%$ and $18.73 \pm 1.53$ vs. $12.13 \pm 2.12 \%$, respectively; $\mathrm{P}=0.038$ and $\mathrm{P}=0.012$ ). In addition, pretreatment with vaspin decreased the percentage of TUNEL-positive cells (Fig. 3B).

Vaspin downregulates the expression of proteins associated with ER stress-induced apoptosis in macrophages. As shown in Fig. 4A and B, ATF6, CHOP, t-JNK1/2, cleaved-caspase 12, cleaved-caspase 9 and cleaved-caspase 3 were overexpressed in response to ox-LDL $(50 \mu \mathrm{g} / \mathrm{ml})$ incubation but were markedly suppressed by vaspin pretreatment $(100 \mathrm{ng} / \mathrm{ml})$. Western blotting was performed to confirm the that vaspin inhibited the progression of atherosclerotic plaques via downregulation of proteins associated with ER stress-induced apoptosis.

\section{Discussion}

The results of the present study demonstrated that vaspin was able to inhibit the progression of atherosclerotic plaques in apoE ${ }^{-/}$mice. The underlying protective mechanism was partly associated with the inhibition of ER stress-induced macrophage apoptosis. Vaspin is well known for its ability to improve insulin sensitivity $(1,2)$. Numerous studies regarding vaspin, including our previous study, have demonstrated that vaspin exerts anti-inflammatory and beneficial effects in cardiovascular disease, in addition to its protective role in metabolic disease $(10,12,22)$. As well as our previous study (10), Phalitakul et al (23) reported that vaspin has an inhibitory role on VSMC inflammation by affecting inflammatory signaling pathways. In addition, Jung et al demonstrated that vaspin may protect vascular endothelial cells against free fatty acid-induced apoptosis via upregulation of the phosphoinositide 3-kinase/Akt signaling pathway (9).

The results of the present study demonstrated that vaspin was able to inhibit ER stress-induced macrophage apoptosis. In addition, vaspin inhibited ER stress and apoptosis in vivo, as detected by reductions in the expression levels of CHOP in the aortic sinus following injection with a vaspin-encoding lentivirus. Notably, total cholesterol, but not serum glucose or lipid 

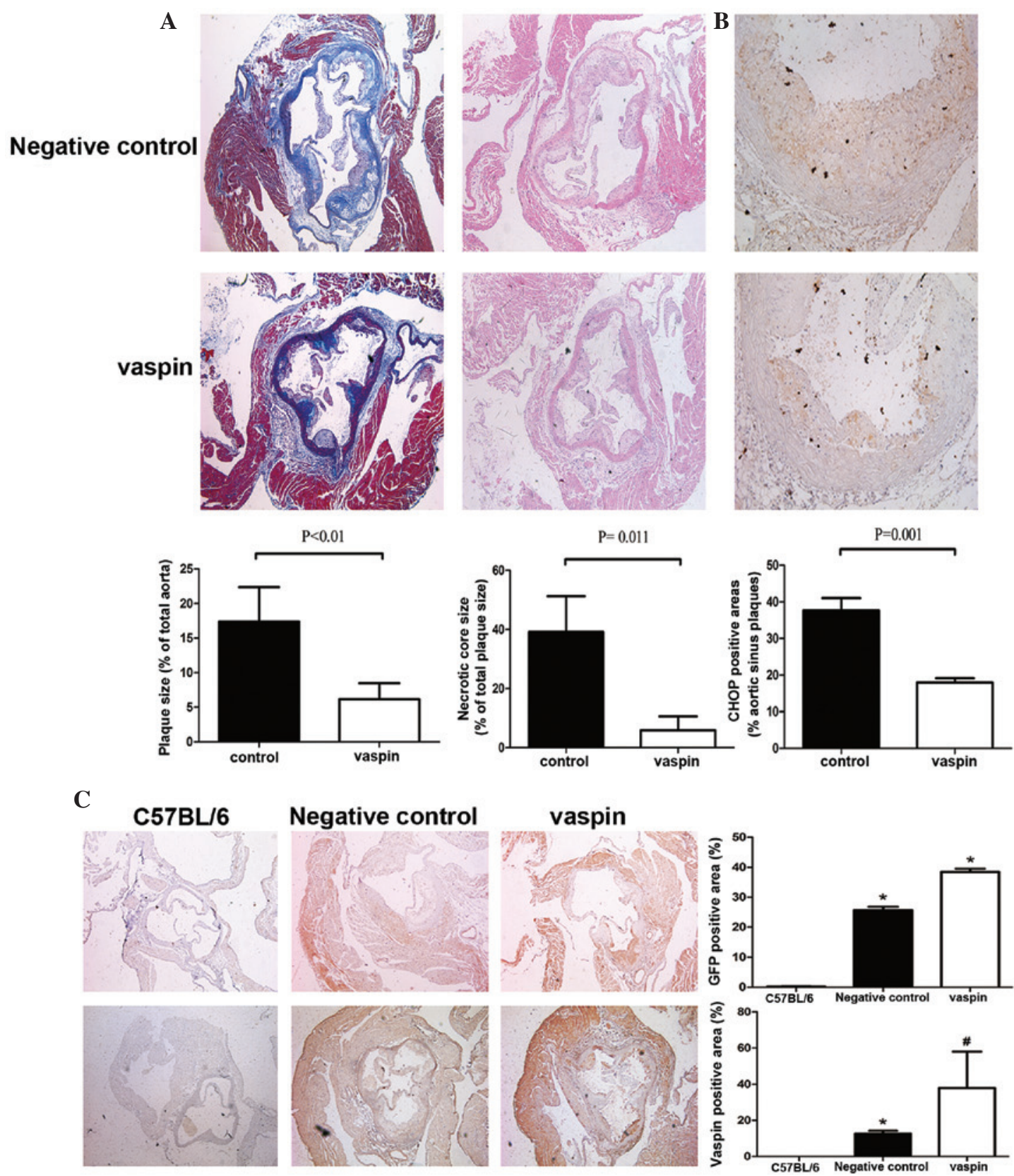

Figure 2. Visceral adipose tissue-derived serine protease inhibitor (vaspin) affects plaque composition in apolipoprotein $\mathrm{E}(\mathrm{apoE})^{-/ /}$mice and attenuates endoplasmic reticulum stress-induced apoptosis in macrophages. (A) Masson's trichrome staining and hematoxylin and eosin staining. Results represent the percentage of plaque size and necrotic core size. Magnification, x50. (B) Immunohistochemical staining of C/EBP-homologous protein (CHOP) in atherosclerotic plaques of the aortic sinus. Brown staining indicates CHOP-positive cells, whereas blue represents hematoxylin counterstaining. Results represent the percentage of areas occupied by CHOP vs. total plaque area within the aortic sinus. Magnification, x200 (C) Transfection efficiency of lentivirus. Results representing the percentage of green fluorescent protein (GFP)-positive areas vs. total plaque area within the aortic sinus, and the percentage of vaspin-positive areas vs. total plaque area within the aortic sinus. Magnification, $\mathrm{x} 50$. Data were obtained from five mice from each group. Data are presented as the mean \pm standard error of the mean. "P $<0.05$ compared with the C57BL/6 mice; ${ }^{~} \mathrm{P}<0.05$ compared with the negative control mice.

levels, was significantly altered by vaspin, thus suggesting that the inhibitory effects of vaspin on lesion growth may be largely independent of lipid profile changes. However, the mechanisms underlying the antiapoptotic effects of vaspin on macrophages remain unclear. Nakatsuka et al $(11,12)$ reported that vaspin is a ligand for the cell surface-associated GRP78/MTJ-1 complex in the liver, and is a novel ligand for the cell surface GRP78/VDAC complex in endothelial cells, promoting proliferation, inhibiting apoptosis, and protecting against diabetes-induced vascular injury. The function of GRP78 is largely associated with the ER of cells. Nakatsuka et al was first to clarify the mechanisms underlying the antiapoptotic action of vaspin in vascular endothelial cells, and implied that vaspin may have a positive role against ER stress.

It is well known that prolonged ER stress leads to cell death via apoptosis, and numerous apoptotic pathways have been identified. One apoptotic pathway is associated with the transcriptional induction of $\mathrm{CHOP}$, which is a member of the $\mathrm{C} / \mathrm{EBP}$ family of transcription factors (24) that can be induced by ATF6 (16). Under normal conditions, CHOP is either not expressed or expressed at low levels; however, it is markedly activated in response to ER stress (25). CHOP overexpression 
A

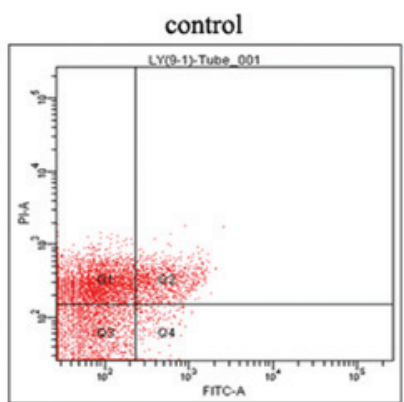

ox-LDL $(50 \mu \mathrm{g} / \mathrm{ml}, 24 \mathrm{~h})+$ vaspin $(100 \mathrm{ng} / \mathrm{ml})$ ox-LDL $(50 \mu \mathrm{g} / \mathrm{ml}, 48 \mathrm{~h})+\mathrm{vaspin}(100 \mathrm{ng} / \mathrm{ml})$
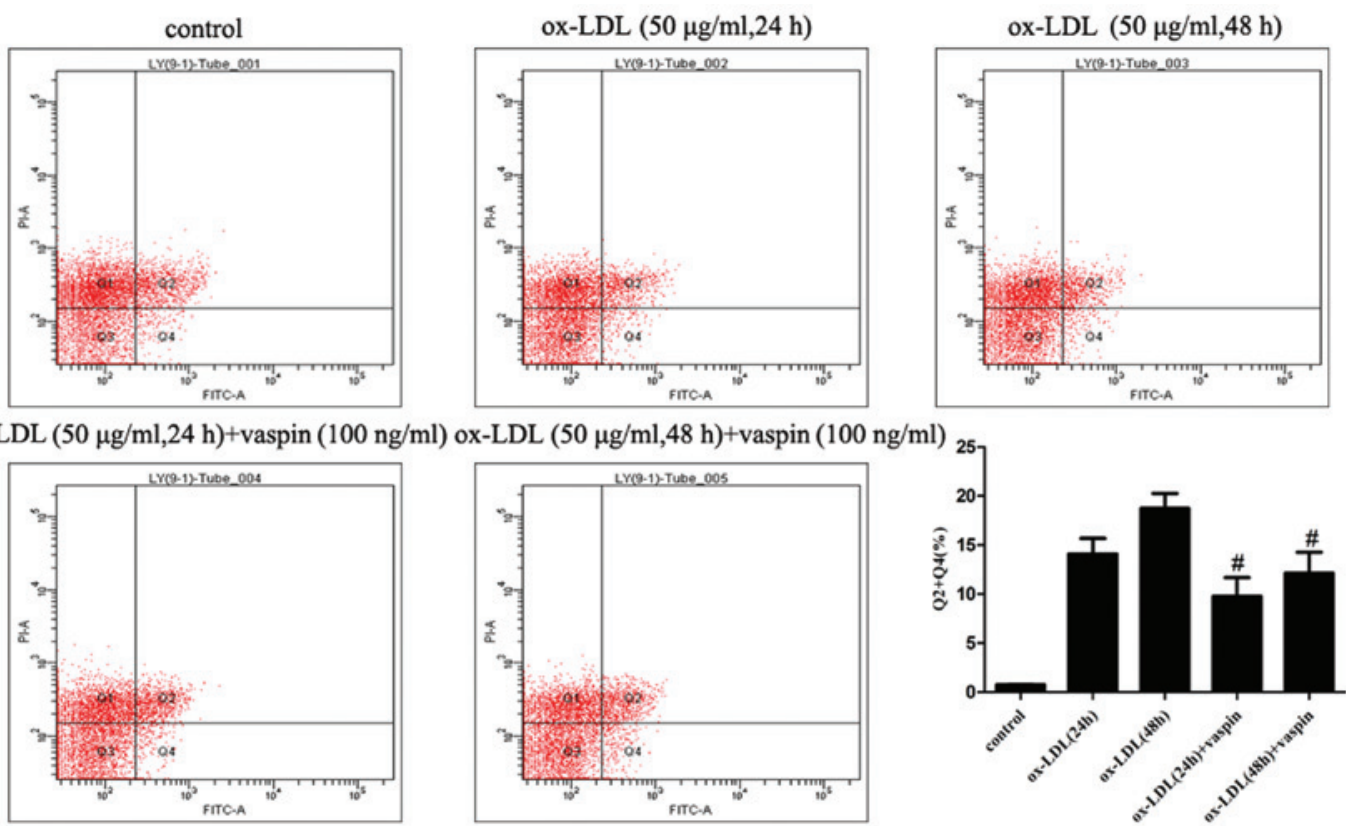

B

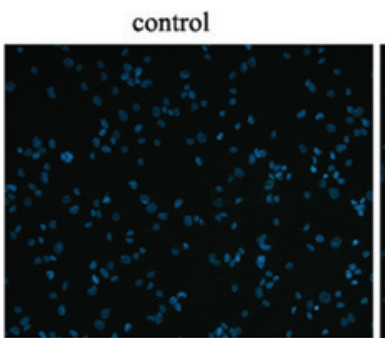

ox-LDL $(50 \mu \mathrm{g} / \mathrm{ml}, 48 \mathrm{~h})$

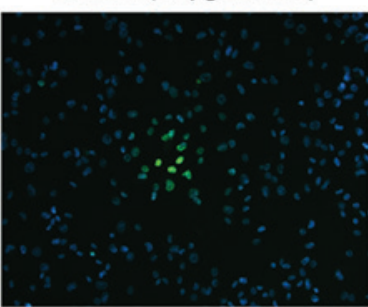

$\operatorname{vaspin}(100 \mathrm{ng} / \mathrm{ml})$
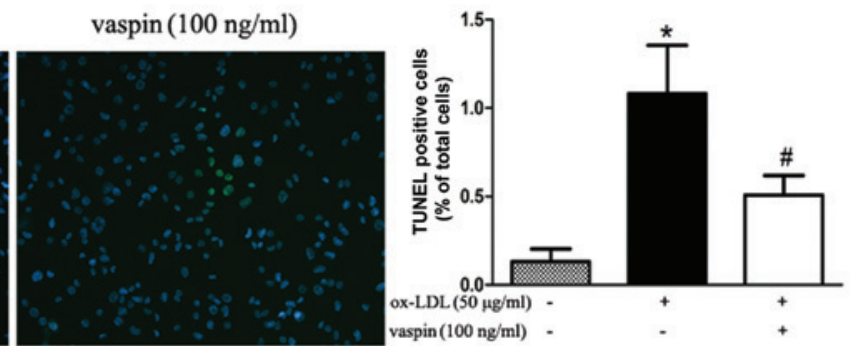

Figure 3. Visceral adipose tissue-derived serine protease inhibitor (vaspin) attenuates endoplasmic reticulum stress-induced apoptosis in macrophages. (A) Flow cytometry dot plots present necrotic and apoptotic cell populations based on Annexin V and propidium iodide (PI) staining. In each plot, the upper left quadrant corresponds to necrotic cells (Annexin V- PI+), the upper right quadrant contains late apoptotic cells (Annexin V+ PI+), the lower left quadrant contains viable cells (Annexin V- PI-), and the lower right quadrant represents early apoptotic cells (Annexin V+ PI-). The rate of apoptosis was quantified by flow cytometry. ${ }^{\#} \mathrm{P}<0.05$ vs. oxidized-low-density lipoprotein (ox-LDL) alone. (B) Percentage of apoptotic THP-1 macrophages, as determined by terminal deoxyribonucleotide transferase-mediated deoxyuridine triphosphate nick-end labeling (TUNEL) assay. Magnification, $x 50$. Data are presented as the mean \pm standard error of the mean from triplicate experiments. ${ }^{*} \mathrm{P}<0.001$ compared with the control cells; ${ }^{*} \mathrm{P}<0.001$ compared with ox-LDL alone.
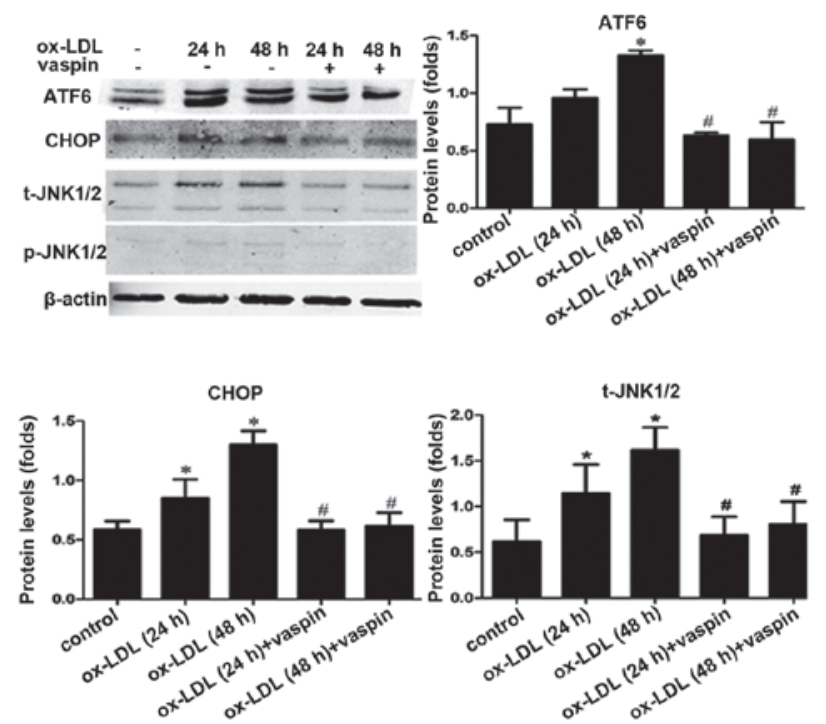

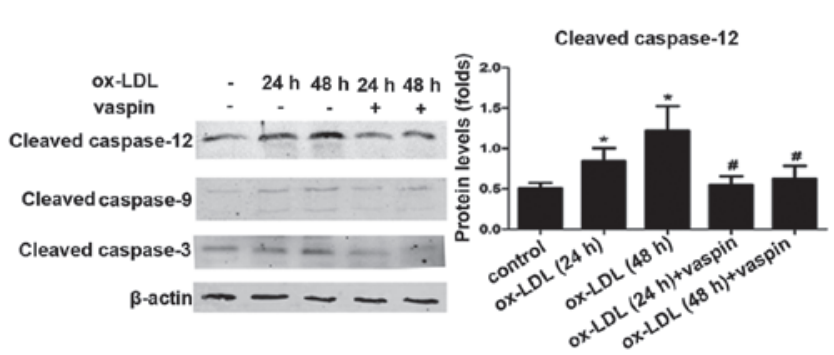

Cleaved caspase-9
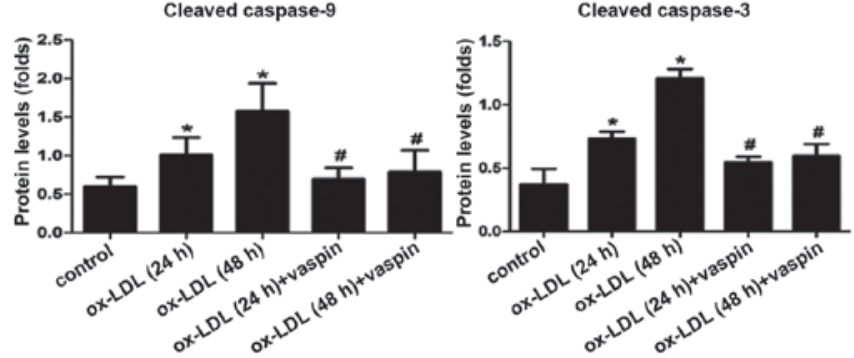

Figure 4. Visceral adipose tissue-derived serine protease inhibitor (vaspin) downregulates the expression levels of proteins associated with endoplasmic reticulum stress-induced apoptosis in macrophages. Activating transcription factor (ATF)6, C/EBP-homologous protein (CHOP), total (t)-c-Jun N-terminal kinases (JNK)1/2, phosphorylated (p)-JNK1/2, cleaved-caspase 12, cleaved-caspase 9, and cleaved-caspase 3 protein expression levels were determined by western blot analysis. Each band density was normalized to control. Data are presented as the mean \pm standard error of the mean. " $\mathrm{P}<0.05$ compared with the control cells; ${ }^{\#} \mathrm{P}<0.05$ compared with oxidized-low-density lipoprotein (ox-LDL) $(50 \mu \mathrm{g} / \mathrm{ml})$ alone. 
can induce apoptosis, and a previous study demonstrated that CHOP expression within atherosclerotic plaques exhibited a strong correlation with the stage of coronary artery lesion, with both parameters markedly increasing in plaques with vulnerable morphology (26). Another pathway is associated with the activation of JNK, which comprises a family of signal transduction proteins that regulate gene expression and participate in decisions regarding apoptosis and survival in response to stress (24). The results of the present study demonstrated that the expression levels of ATF6, CHOP and JNK1/2 were significantly inhibited by vaspin, and $\mathrm{CHOP}$ expression and necrotic area were decreased in the atherosclerotic plaques of vaspin-transfected apoE $\mathrm{E}^{-/-}$mice. These results confirmed that vaspin was able to inhibit the progression of atherosclerosis by suppressing ER stress-induced macrophage apoptosis in apoE ${ }^{-/-}$mice.

Zhou et al demonstrated that macrophages accumulated in atherosclerotic lesions undergoing ER stress, and CHOP expression was increased during the progression of atherosclerosis in chow-fed or Western diet-fed apoE $\mathrm{E}^{-/-}$mice (27). These data indicated that CHOP expression increases as lesions progress, a concept that has been expanded to human atherosclerosis by Myoishi et al (26). In addition, a previous study reported that $\mathrm{CHOP}$ deficiency suppressed atherosclerotic progression in apoE $\mathrm{E}^{-/-}$mice, which is consistent with the results of the present study, and confirmed that the mechanism underlying atherosclerotic inhibition via CHOP deficiency is associated with decreased atherosclerotic plaque necrosis and apoptosis of macrophages (28). CHOP-mediated macrophage apoptosis also contributes to the instability of atherosclerotic plaques, thus suggesting the importance of CHOP in macrophages (29).

ER stress may also lead to apoptosis via activation of caspase 12 (24). Caspase 12 is specifically activated in cells undergoing ER stress. Furthermore, caspase 12-deficient cells are resistant to ER stress inducers, suggesting that caspase-12 is significant in ER stress-induced apoptosis (30). Upon ER stress, procaspase 12 is cleaved and activated, which in turn activates caspase 9/3, thereby leading to cell death (31). The present study demonstrated that the expression levels of caspase 12, caspase 9 and caspase 3 were decreased by vaspin pretreatment. Furthermore, vaspin suppressed ER stress-induced apoptosis, as determined by FCM analysis and TUNEL assay.

In conclusion, the results of the present study indicated that vaspin was able to inhibit the progression of atherosclerotic plaques in apoE ${ }^{-/-}$mice. The potential underlying mechanism is partly associated with the inhibition of ER stress-induced macrophage apoptosis. These findings confirmed that vaspin may have a beneficial role in ameliorating the progression of atherosclerosis, and provide novel insights into the protective function of vaspin in atherosclerosis, thus suggesting that vaspin may be potentially useful for preventing vascular, as well as metabolic, diseases.

\section{Acknowledgements}

The present study was supported by the National Natural Science Foundation of China (grant no. 81270256) to Professor Yawei Xu.

\section{References}

1. Hida K, Wada J, Eguchi J, Zhang H, Baba M, Seida A, Hashimoto I, Okada T, Yasuhara A, Nakatsuka A, et al: Visceral adipose tissue-derived serine protease inhibitor: A unique insulin-sensitizing adipocytokine in obesity. Proc Natl Acad Sci USA 102: 10610-10615, 2005.

2. Wada J: Vaspin: A novel serpin with insulin-sensitizing effects. Expert Opin Investig Drugs 17: 327-333, 2008.

3. Chang HM, Lee HJ, Park HS, Kang JH, Kim KS, Song YS and Jang YJ: Effects of weight reduction on serum vaspin concentrations in obese subjects: Modification by insulin resistance. Obesity (Silver Spring) 18: 2105-2110, 2010.

4. Hida K, Poulsen P, Teshigawara S, Nilsson E, Friedrichsen M, Ribel-Madsen R, Grunnet L, Lund SS, Wada J and Vaag A: Impact of circulating vaspin levels on metabolic variables in elderly twins. Diabetologia 55: 530-532, 2012.

5. Youn BS, Klöting N, Kratzsch J, Lee N, Park JW, Song ES, Ruschke K, Oberbach A, Fasshauer M, Stumvoll M and Blüher M: Serum vaspin concentrations in human obesity and type 2 diabetes. Diabetes 57: 372-377, 2008.

6. Tan BK, Heutling D, Chen J, Farhatullah S, Adya R, Keay SD, Kennedy CR, Lehnert H and Randeva HS: Metformin decreases the adipokine vaspin in overweight women with polycystic ovary syndrome concomitant with improvement in insulin sensitivity and a decrease in insulin resistance. Diabetes 57: 1501-1507, 2008.

7. Seeger J, Ziegelmeier M, Bachmann A, Lössner U, Kratzsch J, Blüher M, Stumvoll M and Fasshauer M: Serum levels of the adipokine vaspin in relation to metabolic and renal parameters. J Clin Endocrinol Metab 93: 247-251, 2008.

8. Li HL, Peng WH, Cui ST, Lei H, Wei YD, Li WM and Xu YW: Vaspin plasma concentrations and mRNA expressions in patients with stable and unstable angina pectoris. Clin Chem Lab Med 49: 1547-1554, 2011.

9. Jung CH, Lee WJ, Hwang JY, Seol SM, Kim YM, Lee YL and Park JY: Vaspin protects vascular endothelial cells against free fatty acid-induced apoptosis through a phosphatidylinositol 3-kinase/Akt pathway. Biochem Biophys Res Commun 413: 264-269, 2011.

10. Li H, Peng W, Zhuang J, Lu Y, Jian W, Wei Y, Li W and Xu Y: Vaspin attenuates high glucose-induced vascular smooth muscle cells proliferation and chemokinesis by inhibiting the MAPK, $\mathrm{PI} 3 \mathrm{~K} / \mathrm{Akt}$ and NF- $\mathrm{KB}$ signaling pathways. Atherosclerosis 228: 61-68, 2013.

11. Nakatsuka A, Wada J, Iseda I, Teshigawara S, Higashio K, Murakami K, Kanzaki M, Inoue K, Terami T, Katayama A, et al: Vaspin is an adipokine ameliorating ER stress in obesity as a ligand for cell-surface GRP78/MTJ-1 complex. Diabetes 61: 2823-2832, 2012

12. Nakatsuka A, Wada J, Iseda I, Teshigawara S, Higashio K, Murakami K, Kanzaki M, Inoue K, Terami T, Katayama A, et al: Visceral adipose tissue-derived serine proteinase inhibitor inhibits apoptosis of endothelial cells as a ligand for the cell-surface GRP78/voltage-dependent anion channel complex. Circ Res 112: 771-780, 2013.

13. Seimon T and Tabas I: Mechanisms and consequences of macrophage apoptosis in atherosclerosis. J Lipid Res 50 (Suppl): S382-S387, 2009.

14. Andrés V, Pello OM and Silvestre-Roig C: Macrophage proliferation and apoptosis in atherosclerosis. Curr Opin Lipidol 23: 429-438, 2012.

15. Hotamisligil GS: Endoplasmic reticulum stress and the inflammatory basis of metabolic disease. Cell 140: 900-917, 2010.

16. Scull CM and Tabas I: Mechanisms of ER stress-induced apoptosis in atherosclerosis. Arterioscler Thromb Vasc Biol 31: 2792-2797, 2011.

17. Aust G, Richter O, Rohm S, Kerner C, Hauss J, Klöting N, Ruschke K, Kovacs P, Youn BS and Blüher M: Vaspin serum concentrations in patients with carotid stenosis. Atherosclerosis 204: 262-266, 2009.

18. Kadoglou NP, Gkontopoulos A, Kapelouzou A, Fotiadis G, Theofilogiannakos EK, Kottas G and Lampropoulos S: Serum levels of vaspin and visfatin in patients with coronary artery disease-Kozani study. Clin Chim Acta 412: 48-52, 2011.

19. Zhang B, Peng W, Li H, Lu Y, Zhuang J, Wang K, Su Y and Xu Y: Plasma vaspin concentrations are decreased in acute coronary syndrome, but unchanged in patients without coronary lesions. Clin Biochem 46: 1520-1525, 2013. 
20. National Research Council (US) Committee for the Update of the Guide for the Care and Use of Laboratory Animals: Guide for the Care and Use of Laboratory Animals. 8th edition. National Institutes of Health, The National Academies Press, Washington (DC), 2011

21. Zhuang J, Peng W, Li H, Lu Y, Wang K, Fan F, Li S and Xu Y: Inhibitory effects of vinpocetine on the progression of atherosclerosis are mediated by Akt/NF-kB dependent mechanisms in apoE ${ }^{-/}$mice. PloS One 8: e82509, 2013

22. Phalitakul S, Okada M, Hara Y and Yamawaki H: A novel adipocytokine, vaspin inhibits platelet-derived growth factor-BB-induced migration of vascular smooth muscle cells. Biochem Biophys Res Commun 423: 844-849, 2012.

23. Phalitakul S, Okada M, Hara Y and Yamawaki H: Vaspin prevents TNF- $\alpha$-induced intracellular adhesion molecule-1 via inhibiting reactive oxygen species-dependent $\mathrm{NF}-\kappa \mathrm{B}$ and $\mathrm{PKC} \theta$ activation in cultured rat vascular smooth muscle cells. Pharmacol Res 64: 493-500, 2011

24. Oyadomari S, Araki E and Mori M: Endoplasmic reticulum stress-mediated apoptosis in pancreatic beta-cells. Apoptosis 7: $335-345,2002$.

25. Wang XZ, Lawson B, Brewer JW, Zinszner H, Sanjay A, Mi LJ, Boorstein R, Kreibich G, Hendershot LM and Ron D: Signals from the stressed endoplasmic reticulum induce C/EBP-homologous protein (CHOP/GADD153). Mol Cell Biol 16: 4273-4280, 1996.
26. Myoishi M, Hao H, Minamino T, Watanabe K, Nishihira K, Hatakeyama K, Asada Y, Okada K, Ishibashi-Ueda H, Gabbiani G, et al: Increased endoplasmic reticulum stress in atherosclerotic plaques associated with acute coronary syndrome. Circulation 116: 1226-1233, 2007.

27. Zhou J, Lhoták S, Hilditch BA and Austin RC: Activation of the unfolded protein response occurs at all stages of atherosclerotic lesion development in apolipoprotein E-deficient mice. Circulation 111: 1814-1821, 2005.

28. Thorp E, Li G, Seimon TA, Kuriakose G, Ron D and Tabas I: Reduced apoptosis and plaque necrosis in advanced atherosclerotic lesions of $\mathrm{Apoe}^{-/-}$and $\mathrm{Ldlr}^{-/}$mice lacking CHOP. Cell Metab 9: 474-481, 2009

29. Tsukano H, Gotoh T, Endo M, Miyata K, Tazume H, Kadomatsu T, Yano M, Iwawaki T, Kohno K, Araki K, et al: The endoplasmic reticulum stress-C/EBP homologous protein pathway-mediated apoptosis in macrophages contributes to the instability of atherosclerotic plaques. Arterioscler Thromb Vasc Biol 30: 1925-1932, 2010.

30. Morishima N, Nakanishi K, Takenouchi H, Shibata T and Yasuhiko Y: An endoplasmic reticulum stress-specific caspase cascade in apoptosis. Cytochrome $c$-independent activation of caspase-9 by caspase-12. J Biol Chem 277: 34287-34294, 2002.

31. Minamino T, Komuro I and Kitakaze M: Endoplasmic reticulum stress as a therapeutic target in cardiovascular disease. Circ Res 107: 1071-1082, 2010. 